\title{
XIII. On Cauchy's and Green's doctrine of extraneous force to explain dynamically Fresnel's kinematics of double refraction
}

\section{Sir William Thomson}

To cite this article: Sir William Thomson (1888) XIII. On Cauchy's and Green's doctrine of extraneous force to explain dynamically Fresnel's kinematics of double refraction, Philosophical Magazine Series 5, 25:153, 116-128, DOI: 10.1080/14786448808628159

To link to this article: http://dx.doi.org/10.1080/14786448808628159

曲 Published online: 29 Apr 2009.

Submit your article to this journal $[\pi$

Џll Article views: 3

Q View related articles $₫$ 
the temperatures of the outside surface and of the inside will after a time become more or less considerable. The outside, being always cooler than the inside, will sooner reach the critical temperature at which there is a sudden increase of thermal conductivity, and, as a consequence, there will be a sudden rush of heat from the inside to the outside; hence the reglow. The assumption that a sudden increase of thermal conductivity should take place when the metal has cooled to a certain temperature is very reasonable; for there is, without doubt, a sudden increase (or, to speak more correctly, more than one* sudden increase) of electrical conductivity as the iron cools.

Mr. H. F. Newall asserts $\dagger$, however, that he has shown that the reglow is "not due to differences in conductivity in iron at different temperatures," and that "there is a rise of temperature not only at the surface ... but also throughout the mass." Mr. Newall has not yet brought before us any experimental evidence in proof of these assertions ; but he has promised to do so.

Mr. Newall seems to agree with the author that the process going on during recalescence "partakes of the nature of an explosion, in that once started it continues throughout the mass of the iron," but he regards the rise of temperature as being caused by internal chemical action. The author looks forward with much interest to the publication of Mr. Newall's results.

XIII. On Cauchy's and Green's Doctrine of Extraneous Force to explain dynamically Fresnel's Kinematics of Double Refraction. By Sir William Thomson. $\ddagger$

1. GREEN'S dynamies of polarization by reflexion, and T Stokes' dynamics of the diffraction of polarized light, and Stokes' and Rayleigh's dynamics of the blue sky, all agree in, as seems to me, irrefragably, demonstrating Fresnel's original conclusion, that in plane polarized light the line of vibration is perpendicular to the plane of polarization; the "plane of polarization" being defined as the plane through the ray and perpendicular to the reflecting surface, when light is polarized by reflexion.

* We should expect, therefore, more than one surface-reheating.

+ Phil. Mag. vol. xxiv. No. 150, p. 436 (1887).

$\mp$ Communicated by the Author, having been read before the Royal Society of Edinburgh, Dec. 5, 1887. 
2. Now when polarized light is transmitted through a crystal, and when rays in any one of the principal planes are examined, it is found that-

(1) A ray with its plane of polarization in the principal plane travels with the same speed, whatever be its direction (whence it is called the "ordinary ray" for that principal plane) ; and (2) A ray whose plane of polarization is perpendicular to the principal plane, and which is called "the extraordinary ray" of that plane, is transmitted with velocity differing for different directions, and having its maximum and minimum values in two mutually perpendicular directions of the ray.

3. Hence and by $\S 1$, the velocities of all rays having their vibrations perpendicular to one principal plane are the same; and the velocities of rays in a principal plane which have their directions of vibration in the same principal plane, differ according to the direction of the ray, and have maximum and minimum values for directions of the ray at right angles to one a nother. But in the laminar shearing or distortional motion of which the wave-motion of the light consists, the "plane of the shear"* (or "plane of the distortion," as it is sometimes called), is the plane through the direction of the ray and the direction of vibration; and therefore it would be the ordinary ray that would have its line of vibration in the principal plane, if the ether's difference of quality in different directions were merely the aeolotropy of an unstrained elastic solid + . Hence ether in a crystal must have something essentially different from mere intrinsic aeolotropy; something that can give different velocities of propagation to two rays, of one of which the line of vibration and line of propagation coincide respectively with the line of propagation and line of vibration of the other.

4. The difficulty of imagining what this something could possibly be, and the utter failure of dynamics to account for double refraction without it, have been generally felt to be the greatest imperfection of optical theory.

It is true that ever since 1839 a suggested explanation bas been before the world; given independently by Cauchy and Green, in what Stokes has called their "Second Theories of Double Refraction," presented on the same day, the 20th of

* Thomson and Tait's 'Natural Philosophy,' $\$ 171$ (or 'Elements, $\S 150)$.

+ The elementary dynamics of elastic solids, shows that on this supposition there might be maximum and minimum velocities of propagation for rays in directions at $45^{\circ}$ to one another, but that the velocities must essentially be equal for every two directions at $90^{\circ}$ to one another, in the principal plane, when the line of vibration is in this plane. 
May of that year, to the French Academy of Sciences and the Cambridge Philosophical Society. Stokes, in his Report on Double Refraction, ${ }^{*}$ has given a perfectly clear account of this explanation. It has been but little noticed otherwise, and somehow it has not been found generally acceptable ; perhaps, because of a certain appearance of artificiality and arbitrariness of assumption which might be supposed to discredit it. But whatever may have been the reason or reasons which have caused it to be neglected as it has been, and though it is undoubtedly faulty, both as given by Cauchy and by Green, it contains what seems to me, in all probability, the true principle of the explanation, and which is, that the ether in a doubly refracting crystal is an elastic solid, nnequally pressed or unequally pulled in different directions, by the unmoved ponderable matter.

5. Cauchy's work on the wave-theory of light is complicated throughout, and to some degree vitiated, by admission of the Navier-Poisson false doctrine $\dagger$ that compressibility is calculable theoretically from rigidity ; a doctrine which Green sets aside, rightly and conveniently, by simply assuming incompressibility. In other respects Cauchy's and Green's "Second Theories of Double Refraction" as Stokes calls them, are almost identical. Each supposes ether in the crystal to be an intrinsically aeolotropic elastic solid, having its aelotropy modified in virtue of internal pressure or pull, equal or unequal in different directions, produced by and balanced by extraneous force. Each is faulty in leaving intrinsic rigidity-moduluses (coefficients) unaffected by the equilibrium-pressure, and in introducing three fresh terms, with coefficients (A, B, C in Green's notation) to represent the whole effect of the equilibrium-pressure. This gives for the case of an intrinsically isotropic solid, augmentation of virtual rigidity, and therefore of wave-velocity, by equal pull $\ddagger$ in all directions, and diminution by equal positive pressure in all directions; which is obviously wrong. 'Thus definitively, pull in all directions outwards perpendicular to the bounding

* British Association Report, 1862.

$\dagger$ See Stokes, 'On the Friction of Fluids in Motion and on the Equilibrium and Motion of Elastic Solids,' Camb. Phil. Trans., 1845, $\$$ 19, 20 ; reprinted in Stokes 'Mathematical and Physical Papers,' vol. i. p. 123; or Thomson and Tait's ' Natural Philosophy,' $\$ \$ 684,685$; or 'Elements,' $\$ \$ 655,656$.

$\ddagger$ So little has been done towards interpreting the formulas of either writer that it has not been hitherto noticed that positive values of Cauchy's G, H, I, or of Green's A, B, C, signify pulls, and negative values signify pressures. 
surface equal per unit of area to three times the intrinsic rigidity-modulus, would give quadrupled virtual rigidity, and therefore doubled wave-velocity! Positive normal pressure inwards equal to the intrinsic rigidity-modulus would annul the rigidity and the wave-velocity - that is to say, would make a fluid of the solid. And, on the other hand, negative pressure, or outward pull, on an incompressible liquid, would give it virtual rigidity, and render it capable of transmitting laminar waves! It is obvious that abstract dynamics can show for pressure or pull equal in all directions, no effect on any physical property of an incompressible solid or fluid.

6. Again, pull or pressure unequal in different directions, on an isotropic incompressible solid, would, according to Green's formula (A) in p. 303 of his collected Mathematical Papers, cause the velocity of a laminar wave to depend simply on the wave-front, and to have maximum, minimax, and minimum velocities for wave-fronts perpendicular respectively to the directions of maximum pull, minimax pull, and minimum pull; and would make the wave-surface a simple ellipsoid! This, which would be precisely the case of foam stretched unequally in different directions, seemed to me a very interesting and important result, until (as shown in $\$ 19$ below) $I$ found it to be not true.

7. To understand fully the stress-theory of double refraction, we may help ourselves effectively by working out directly and thoroughly (as is obviously to be done quite easily by abstract dynamics) the problem of $\$ 6$, as follows :-Suppose the solid, isotropic when unstrained, to become strained by pressure so applied to its boundary as to produce, throughout the interior, homogeneous strain according to the following specification :-

The coordinates of any point $M$ of the mass which were $\xi, \eta, \zeta$ when there was no strain, become in the strained solid

$$
\xi \sqrt{ } \alpha, \eta \vee \beta, \zeta \vee \gamma \ldots . . .
$$

$\sqrt{ } \alpha, \sqrt{ } \beta, \sqrt{ } \gamma$, or the "Principal Elongations" *, being the same whatever point $M$ of the solid we choose. Because of incompressibility we have

$$
\alpha \beta \gamma=1 \text {. . . . . . }
$$

"See chap. iv. of "Mathematical Theory of Elasticity" (W. Thomson), Trans. Roy. Soc. Lond. 1856, reprinted in vol. iii. of 'Mathematical and Physical.Payers,' now on the point of being published, or Thomson and Tait's 'Natural Philosophy,' $\$ 160,164$, or 'Elements,' \$\$ 141, 158. 
For brevity, we shall designate as $(\alpha, \beta, \gamma)$ the strained condition thus defined.

8. As a purely kinematic preliminary, let it be required to find the principal strain-ratios when the solid, already strained according to (1), (2), is further strained by a uniform shear, $\sigma$, specified as tollows; in terms of $x, y, z$, the coordinates of still the same particle, M, of the solid and other notation, as explained below :-

where

$$
\left.\begin{array}{l}
x=\xi \sqrt{ } \alpha+\sigma p l \\
y=\eta \sqrt{ } \beta+\sigma p m \\
z=\zeta \sqrt{ } \gamma+\sigma p n
\end{array}\right\} . . . . .
$$

with

$$
p=\mathrm{OP}=\lambda \xi \sqrt{ } \alpha+\mu \eta \sqrt{ } \beta+\nu \zeta \sqrt{ } \gamma \text {. . . }
$$

and

$$
l^{2}+m^{2}+n^{2}=1, \quad \lambda^{2}+\mu^{2}+\nu^{2}=1 \quad \text {. . . (5), }
$$

$$
l \lambda+m \mu+n \nu=0 \text {. . . . . . (6); }
$$

$\lambda, \mu, \nu$ denoting the direction-cosines of $\mathrm{OP}$, the normal to the shearing planes, and $l, m, n$ the direction-cosines of shearing displacement. The principal axes of the resultant strains are the directions of $O M$ in which it is maximum or minimum, subject to the condition

$$
\xi^{2}+\eta^{2}+\zeta^{2}=1
$$

and its maximum, minimax, and minimum values are the three required strain-ratios. Now we have

$$
\begin{aligned}
\mathrm{OM}^{2} & =x^{2}+y^{2}+z^{2} \\
& =\xi^{2} \alpha+\eta^{2} \beta+\zeta^{2} \gamma+2 \sigma(l \xi \sqrt{ } \alpha+m \eta \sqrt{ } \beta+n \zeta \sqrt{ } \gamma) p+\sigma^{2} p^{2}
\end{aligned}
$$

and to make this maximum or minimum subject to $(7)$, we have

$$
\frac{d\left(\frac{1}{2} O M^{2}\right)}{d \xi}=\rho \xi ; \frac{d\left(\frac{1}{2} O M^{2}\right)}{d \eta}=\rho \eta ; \frac{d\left(\frac{1}{2} O M^{2}\right)}{d \zeta}=\rho \zeta .
$$

where in virtue of (7), and because $O M^{2}$ is a homogeneous quadratic function of $\xi, \eta, \zeta$,

$$
\rho=\mathrm{OM}^{2} \text {. }
$$

The determinantal cubic, being

$$
(\mathscr{A}-\rho)(\mathscr{B}-\rho)(\mathscr{G}-\rho)-a^{2}(\mathscr{A}-\rho)-b^{2}(\mathscr{B}-\rho)-c^{2}(\mathscr{C}-\rho)+2 a b c=0(
$$

where

$$
\begin{array}{r}
\mathscr{A}=\alpha\left(1+2 \sigma l \lambda+\sigma^{2} \lambda^{2}\right) ; \mathscr{B}=\beta\left(1+2 \sigma m \mu+\sigma^{2} \mu^{2}\right) ; \\
G=\gamma\left(1+2 \sigma n \nu+\sigma^{2} \nu^{2}\right) . . . . . .
\end{array}
$$


and

$$
\begin{array}{r}
a=\sqrt{ }(\beta \gamma)\left[\sigma(m \nu+n \mu)+\sigma^{2} \mu \nu\right] ; b=\sqrt{ }(\gamma \alpha)\left[\sigma(n \lambda+l \nu)+\sigma^{2} \nu \lambda\right] \\
c=\sqrt{ }(\alpha \beta)\left[\sigma(l \mu+m \lambda)+\sigma^{2} \lambda \mu\right] \quad . \quad \cdot \quad(12),
\end{array}
$$

gives three real positive values for $\rho$, the square roots of which are the required principal strain-ratios.

9. Entering now on the dynamics of our subject, remark that the isotropy ( $\S 1$ ), implies that the work required of the extraneous pressure, to change the solid from its unstrained condition $(1,1,1)$ to the strain $(\alpha, \beta, \gamma)$, is independent of the direction of the normal axes of the strain, and depends solely on the magnitudes of $\alpha, \beta, \gamma$. Hence if $\mathrm{E}$ denotes its magnitude per unit of volume; or the potential energy of unit volume in the condition $(\alpha, \beta, \gamma)$ reckoned from zero in the condition $(1,1,1)$; we have

$$
\mathrm{E}=\psi(\alpha, \beta, \gamma)
$$

where $\psi$ denotes a function of which the magnitude is unaltered when the values of $\alpha, \beta, \gamma$ are interchanged. Consider a portion of the solid, which, in the unstrained condition, is a cube of unit side, and which in the strained condition $(\alpha, \beta, \gamma)$, is a rectangular parallelepiped $\sqrt{ } \alpha \cdot \sqrt{ } \beta \cdot \sqrt{ } \gamma$. In virtue of isotropy and symmetry, we see that the pull or pressure on each of the six faces of this figure, required to keep the substance in the condition $(\alpha, \beta, \gamma)$ is normal to the face. Let the amounts of these forces per unit area, on the three pairs of faces respectively, be A, B, C, each reckoned as positive or negative according as the force is positive pull, or positive pressure. We shall take

$$
\mathrm{A}+\mathrm{B}+\mathrm{C}=0 \text {. . . . . . }
$$

because normal pull or pressure uniform in all directions produces no effect, the solid being incompressible. The work done on any infinitesimal change from the configuration $(\alpha, \beta, \gamma)$ is

$$
\mathrm{A} \sqrt{ }(\beta \gamma) d(\sqrt{ } \alpha)+\mathrm{B} \sqrt{ }(\gamma \alpha) d(\sqrt{ } \beta)+\mathrm{C} \sqrt{ }(\alpha \beta) d(\sqrt{ } \gamma)
$$
or (because $\alpha \beta \gamma=1$ )

$$
\frac{\mathrm{A}}{2 \alpha} d \alpha+\frac{\mathrm{B}}{2 \beta} d \beta+\frac{\mathrm{C}}{2 \gamma} d \gamma
$$

10. Let $\delta \alpha, \delta \beta, \delta \gamma$ be any variations of $\alpha, \beta, \gamma$ consistent with (2), so that we have

and

$$
\left.\begin{array}{c}
(\alpha+\delta \alpha)(\beta+\delta \beta)(\gamma+\delta \gamma)=1 \\
\alpha \beta \gamma=1
\end{array}\right\} \cdot \cdot(16) .
$$

Now suppose $\delta \alpha, \delta \beta, \delta \gamma$ to be so small that we may neglect 
their cubes and corresponding products, and all higher products. We have

whence

$$
\frac{\delta \alpha}{\alpha}+\frac{\delta \beta}{\beta}+\frac{\delta \gamma}{\gamma}+\alpha \delta \beta \delta \gamma+\beta \delta \gamma \delta \alpha+\gamma \delta \alpha \delta \beta=0 \text {. }
$$

$$
\left(\frac{\delta \alpha}{\alpha}\right)^{2}=\left(\frac{\delta \beta}{\beta}+\frac{\delta \gamma}{\gamma}\right)^{2}
$$

whence, and by the symmetrical expressions,

$$
\left.\begin{array}{l}
2 \delta \beta \delta \gamma=\frac{1}{\alpha}\left(\frac{\delta \alpha^{2}}{\alpha^{2}}-\frac{\delta \beta^{2}}{\beta^{2}}-\frac{\delta \gamma^{2}}{\gamma^{2}}\right) \\
2 \delta \gamma \delta \alpha=\frac{1}{\beta}\left(\frac{\delta \beta^{2}}{\beta^{2}}-\frac{\gamma^{2}}{\gamma^{2}}-\frac{\delta \alpha^{2}}{\alpha^{2}}\right) \\
2 \delta \alpha \delta \beta=\frac{1}{\gamma}\left(\frac{\delta \gamma^{2}}{\gamma^{2}}-\frac{\delta \alpha^{2}}{\alpha^{2}}-\frac{\delta \beta^{2}}{\beta^{2}}\right)
\end{array}\right\}
$$

11. Now, if $\mathrm{E}+\delta \mathrm{E}$ denote the energy per unit bulk of the solid in the condition

$$
(\alpha+\delta \alpha, \beta+\delta \beta, \gamma+\delta \gamma)
$$

we have, by Taylor's theorem,

$$
\delta \mathrm{E}=\mathrm{H}_{1}+\mathrm{H}_{2}+\mathrm{H}_{3}+\& c .
$$

where $\mathrm{H}_{1}, \mathrm{H}_{2}$, \&c. denote homogeneous functions of $\delta \alpha, \delta \beta, \delta \gamma$ of the 1st degree, 2nd degree, \&c. Hence, omitting cubes \&c., and eliminating the products from $\mathrm{H}_{2}$, and taking $\mathrm{H}_{1}$ from (15), we find

$$
\delta \mathrm{E}=\frac{1}{2}\left(\frac{\mathrm{A}}{\alpha} \delta \alpha+\frac{\mathrm{B}}{\beta} \delta \beta+\frac{\mathrm{C}}{\gamma} \delta \gamma+\mathrm{G} \frac{\delta \alpha^{2}}{\alpha^{2}}+\mathrm{H} \frac{\delta \beta^{2}}{\beta^{2}}+\mathrm{I} \frac{\delta \gamma^{2}}{\gamma^{2}}\right) .
$$

where $\mathrm{G}, \mathrm{H}, \mathrm{I}$ denote three coefficients depending on the nature of the function $\psi$ (13), which expresses the energy. Thus in (19), with (14) taken into account, we have just five coefficients independently disposable, $\mathrm{A}, \mathrm{B}, \mathrm{G}, \mathrm{H}$, I, which is the right number because, in virtue of $\alpha \beta \gamma=1, \mathrm{E}$ is a function of just two independent variables.

12. For the case of $\alpha=1, \beta=1, \gamma=1$, we have

$$
\mathrm{A}=\mathrm{B}=\mathrm{C}=0 \text { and } \mathrm{G}=\mathrm{H}=\mathrm{I}=\mathrm{G}_{1} \text {, suppose; }
$$

which give

$$
\delta \mathrm{E}=\frac{1}{2} \mathrm{G}_{1}\left(\delta \alpha^{2}+\delta \beta^{2}+\delta \gamma^{2}\right) .
$$

From this we see that $2 G_{1}$ is simply the rigidity-modulus of the unstrained solid; because if we make $\delta \gamma=0$, we have $\delta \alpha=-\delta \beta$, and the strain becomes an infinitesimal distortion 
in the plane (xy), which may be regarded in two ways as a simple shear of which the magnitude is $\delta a^{*}$ (this being twice the elongation in one of the normal axes).

13. Going back to (10), (11), and (12), let $\sigma$ be so small that $\sigma^{3}$ and higher powers can be neglected. To this degree of approximation we neglect $a b c$ in (10), and see that its three roots are respectively

$$
\mathscr{A}-\frac{b^{2}}{\mathscr{C}-\mathscr{A}}-\frac{c^{2}}{\mathscr{B}-\mathscr{A}} ; \quad \mathscr{B}-\frac{c^{2}}{\mathscr{A}-\mathscr{B}}-\frac{a^{2}}{6^{2}-\mathscr{B}} ; \quad \mathscr{C}-\frac{a^{2}}{\mathscr{B}-6}-\frac{b^{2}}{\mathscr{A}-6^{7}}
$$

provided none of the differences constituting the denominators is infinitely small. The case of any of these differences infinitely small, or zero, does not, as we shall see in the conclusion, require special treatment, though special treatment would be needed to interpret for any such case each step of the process.

14. Substituting now for $\mathscr{A}, \mathscr{B}, 6, a, b, o$ in (20), their values by (11) and (12), neglecting $\sigma^{3}$ and higher powers, and denoting by $\delta \alpha, \delta \beta, \delta \gamma$ the excesses of the three roots above $\alpha, \beta, \gamma$ respectively, we find

$$
\left.\begin{array}{l}
\delta \alpha=\alpha\left\{2 \sigma l \lambda+\sigma^{2}\left[\lambda^{2}-\frac{\gamma}{\gamma-\alpha}(n \lambda+l \nu)^{2}-\frac{\beta}{\beta-\alpha}(l \mu+m \lambda)^{2}\right]\right\} \\
\delta \beta=\beta\left\{2 \sigma m \mu+\sigma^{2}\left[\mu^{2}-\frac{\alpha}{\alpha-\beta}(l \mu+m \lambda)^{2}-\frac{\gamma}{\gamma-\beta}(m \nu+n \mu)^{2}\right]\right\} \\
\delta \gamma=\gamma\left\{2 \sigma n \nu+\sigma^{2}\left[\nu^{2}-\frac{\beta}{\beta-\gamma}(m \nu+n \mu)^{2}-\frac{\alpha}{\alpha-\gamma}(n \lambda+l \nu)^{2}\right]\right\}
\end{array}\right\}
$$

and using these in (19), we find

$$
\begin{gathered}
\delta \mathrm{E}=\sigma(\mathrm{A} l \lambda+\mathrm{B} m \mu+\mathrm{C} n \nu) \\
+\frac{1}{2} \sigma^{2}\left\{\mathrm{~A} \lambda^{2}+\mathrm{B} \mu^{2}+\mathrm{C} \nu^{2}+\mathrm{L}(m \nu+n \mu)^{2}+\mathrm{M}(n \lambda+l \nu)^{2}+\mathrm{N}(l \mu+m \lambda)^{2}\right\} \\
+2 \sigma^{2}\left(\mathrm{G} l^{2} \lambda^{2}+\mathrm{H} m^{2} \mu^{2}+\mathrm{I} n^{2} \nu^{2}\right)
\end{gathered}
$$

where

$$
\mathrm{L}=\frac{\mathrm{B} \gamma-\mathrm{C} \beta}{\beta-\gamma} ; \quad \mathrm{M}=\frac{\mathrm{C} \alpha-\mathrm{A} \gamma}{\gamma-\alpha} ; \quad \mathrm{N}=\frac{\mathrm{A} \beta-\mathrm{B} \alpha}{\alpha-\beta} .
$$

15. Now from (5) and (6) we find

$$
(m \nu+n \mu)^{2}=1-l^{2}-\lambda^{2}+2\left(l^{2} \lambda^{2}-m^{2} \mu^{2}-n^{2} \nu^{2}\right) .
$$

which, with the symmetrical expressions, reduces (22) to $\S 154$. 


$$
\begin{aligned}
\delta \mathrm{E} & =\sigma(\mathrm{A} l \lambda+\mathrm{B} m \mu+\mathrm{C} n \nu)+\frac{1}{2} \sigma^{2}\left\{\mathrm{~L}+\mathrm{M}+\mathrm{N}+(\mathrm{A}-\mathrm{L}) \lambda^{2}\right. \\
& +(\mathrm{B}-\mathrm{M}) \mu^{2}+(\mathrm{C}-\mathrm{N}) \nu^{2}-\mathrm{L} l^{2}-\mathrm{M} m^{2}-\mathrm{N} n^{2}+2\left[(2 \mathrm{G}+\mathrm{L}-\mathrm{M}-\mathrm{N}) l^{2} \lambda^{2}\right\} \\
& \left.\left.+(2 \mathrm{H}+\mathrm{M}-\mathrm{N}-\mathrm{L}) m^{2} \mu^{2}+(2 \mathrm{I}+\mathrm{N}-\mathrm{L}-\mathrm{M}) n^{2} v^{2}\right]\right\}
\end{aligned}
$$

16. To interpret this result statically, imagine the solid to be given in the state of bomogeneous strain $(\alpha, \beta, \gamma)$ throughout, and let a finite plane plate of it, of thickness $h$, and of very large area $Q$, be displaced by a shearing motion according to the specification (3), (4), (5), (6) of $\$ 8$; the bounding-planes of the plate being unmoved, and all the solid exterior to the plate being therefore undisturbed except by the slight distortion round the edge of the plate produced by the displacement of its substance. The analytical expression of this is

$$
\sigma=f(p) \quad \cdot \quad \cdot \quad \cdot \quad \cdot
$$

where $f$ denotes any function of OP such that

$$
\int_{0}^{h} d p f(p)=0 \quad \cdot \quad \cdot \quad \cdot \quad .
$$

If we denote by $W$ the work required to produce the supposed displacement, we have

$$
\mathrm{W}=\mathrm{Q} \int_{0}^{h} d p \delta \mathrm{E}+\mathscr{W} \cdot \cdot \cdot .
$$

$\delta \mathrm{E}$ being given by (25), with everything constant except $\sigma$, a function of $O P$; and $\mathscr{W}$ denoting the work done on the solid outside the boundary of the plate. In this expression the first line of (25) disappears in virtue of (27); and we have

$$
\left.\begin{array}{l}
\frac{\mathrm{W}-\mathscr{W}}{\mathrm{Q}}=\frac{1}{2}\left\{\mathrm{~L}+\mathrm{M}+\mathrm{N}+(\mathrm{A}-\mathrm{L}) \lambda^{2}+(\mathrm{B}-\mathrm{M}) \mu^{2}+(\mathrm{C}-\mathrm{N}) \nu^{2}\right. \\
\quad-\mathrm{L} \ell^{2}-\mathrm{M} m^{2}-\mathrm{N} n^{2}+2\left[(2 \mathrm{G}+\mathrm{L}-\mathrm{M}-\mathrm{N}) l^{2} \lambda^{2}\right. \\
\left.\left.\quad+(2 \mathrm{H}+\mathrm{M}-\mathrm{N}-\mathrm{L}) m^{2} \mu^{2}+(2 \mathrm{I}+\mathrm{N}-\mathrm{L}-\mathrm{M}) n^{2} \nu^{2}\right]\right\} \int_{0}^{h} d p \sigma^{2}
\end{array}\right\}
$$

When every diameter of the plate is infinitely great in comparison with its thickness, $\mathscr{U} / \mathrm{Q}$ is infinitely small ; and the second member of (29) expresses the work per unit of area of the plate, required to produce the supposed shearing motion.

17. Solve now the problem of finding, subject to (5) and (6) of $\S 8$, the values of $l, m, n$ which make the factor $\{1$ of the second member of (29) a maximum or minimum. This is only the problem of finding the two principal diameters of the ellipse in which the ellipsoid 


$$
\begin{array}{r}
{\left[2(2 \mathrm{G}+\mathrm{L}-\mathrm{M}-\mathrm{N}) \lambda^{2}-\mathrm{L}\right] x^{2}+\left[2(2 \mathrm{H}+\mathrm{M}-\mathrm{N}-\mathrm{L}) \mu^{2}-\mathrm{M}\right] y^{2}} \\
+\left[2(2 \mathrm{I}+\mathrm{N}-\mathrm{L}-\mathrm{M}) v^{2}-\mathrm{N}\right] z^{2}=\text { const. } .
\end{array}
$$

is cut by the plane

$$
\lambda x+\mu y+\nu z=0 \quad . \quad \cdot \quad .
$$

If the displacement is in either of the two directions $(l, m, n)$ thus determined, the force required to maintain it is in the direction of displacement; and the magnitude of this force per unit bulk of the material of the plate at any point within it is easily proved to be

$$
\{\mathrm{M}\} \frac{d \sigma}{d \rho} \cdot \cdot \cdot \cdot \cdot \cdot \cdot \cdot
$$

where $\{M\}$ denotes the maximum or the minimum value of the bracketed factor of (29).

18. Passing now from equilibrium to motion, we see at once that (the density being taken as unity)

$$
\mathrm{V}^{2}=\{\mathrm{M}\} \quad \cdot \quad \cdot \cdot \cdot \cdot
$$

where $\mathrm{V}$ denotes the velocity of either of two simple waves whose wave-front is perpendicular to $(\lambda, \mu, \nu)$. Consider the case of wave-front perpendicular to on $€$ of the three principal planes ; $(y z)$ for instance : we have $\lambda=0$; and, to make \{\} of (29) a maximum or minimum, we see by symmetry that we must either have

$\left.\begin{array}{l}\text { (vibration perpendicular to principal plane) } l=1, m=0, n=0 \\ \text { (vibration in principal plane) } \ldots . \ldots . \ldots . . . . l=0, m=-\nu, n=\mu\end{array}\right\}$

Hence, for the two cases, we have respectively:

Vibration perpendicular to $y z \quad \ldots V^{2}=\mathrm{M}+\mathrm{N}+(\mathrm{B}-\mathrm{M}) \mu^{2}+(\mathrm{C}-\mathrm{N}) \nu^{2}$

Vibration in $y z \quad$................ $\mathrm{V}^{2}=\mathrm{L}+\mathrm{B} \mu^{2}+\mathrm{C} \nu^{2}+4(\mathrm{H}+\mathrm{I}-\mathrm{L}) \mu^{2} \nu^{2}$ ，

19. According to Fresnel's theory (35) must be constant, and the last term of (36) must vanish. These and the corresponding conclusions relatively to the other two principal planes are satisfied if, and require that,

and

$$
\mathrm{A}-\mathrm{L}=\mathrm{B}-\mathrm{M}=\mathrm{C}-\mathrm{N} \cdot \text {. } .(37),
$$

$$
\mathrm{H}+\mathrm{I}=\mathrm{L} ; \quad \mathrm{I}+\mathrm{G}=\mathrm{M} ; \mathrm{G}+\mathrm{H}=\mathrm{N} \quad \text { (38). }
$$

Transposing $\mathbf{M}$ and $\mathrm{N}$ in the last of equations (37), substituting for them their values by (23), and dividing each member by 
$\beta \gamma$, we find

$$
\frac{\mathrm{C}-\mathrm{A}}{\beta \gamma-\alpha \beta}=\frac{\mathrm{A}-\mathrm{B}}{\gamma \alpha-\beta \gamma} \text {. . . . . }
$$

whence (sum of numerators divided by sum of denominators),

$$
\frac{\mathrm{B}-\mathrm{C}}{\gamma \alpha-\alpha \beta}=\frac{\mathrm{C}-\mathrm{A}}{\alpha \beta-\beta \gamma}=\frac{\mathrm{A}-\mathrm{B}}{\beta \gamma-\gamma \alpha} . .
$$

The first of these equations is equivalent to the first of (37); and thus we see that the two equations (37) are equivalent to one only ; and (39) is a convenient form of this one. By it, as put symmetrically in (40), and by bringing (14) into account, we find, with $k$ taken to denote a coefficient which may be any function of $(\alpha, \beta, \gamma)$ :

$$
\left.\begin{array}{ll}
\mathrm{A}=k\left(\mathrm{~S}-\beta_{\gamma}\right) ; & \mathrm{B}=k(\mathrm{~S}-\gamma \alpha) ; \quad \mathrm{C}=k(\mathrm{~S}-\alpha \beta) ; \\
\mathrm{S}=\frac{1}{3}(\beta \gamma+\gamma \alpha+\alpha \beta)
\end{array}\right\}
$$

and using this result in (23), we find

$$
\left.\begin{array}{l}
\mathrm{L}=k[\alpha(\beta+\gamma)-\mathrm{S}] ; \mathrm{M}=k[\beta(\gamma+\alpha)-\mathrm{S}] ; \mathrm{N}=k[\gamma(\alpha+\beta)-\mathrm{S}] \\
\text { or } \mathrm{L}=k(2 \mathrm{~S}-\beta \gamma) ; \mathrm{M}=k(2 \mathrm{~S}-\gamma \alpha) ; \mathrm{N}=k(2 \mathrm{~S}-\alpha \beta)
\end{array}\right\}
$$

By (2) we may put (41) and (42) into forms more convenient for some purposes as follows :-

where

$$
\begin{aligned}
& \mathrm{A}=k\left(\mathrm{~S}-\frac{1}{\alpha}\right) ; \quad \mathrm{B}=k\left(\mathrm{~S}-\frac{1}{\beta}\right) ; \quad \mathrm{C}=k\left(\mathrm{~S}-\frac{1}{\gamma}\right) . \\
& \mathrm{L}=k\left(2 \mathrm{~S}-\frac{1}{\alpha}\right) ; \mathrm{M}=k\left(2 \mathrm{~S}-\frac{1}{\beta}\right) ; \mathrm{N}=k\left(2 \mathrm{~S}-\frac{1}{\gamma}\right) .
\end{aligned}
$$

$$
\mathrm{S}=\frac{1}{3}\left(\frac{1}{\alpha}+\frac{1}{\beta}+\frac{1}{\gamma}\right) \text {. . . . . }
$$

Next, we find $G, H, I$; by $(38),(44)$, and (45) we have

$$
\mathrm{G}+\mathrm{H}+\mathrm{I}=\frac{1}{2}(\mathrm{~L}+\mathrm{M}+\mathrm{N})=\frac{3}{2} k \mathrm{~S}=\frac{1}{2} k\left(\frac{1}{\alpha}+\frac{1}{\beta}+\frac{1}{\gamma}\right) .
$$

whence, by (38) and (44),

$$
\mathrm{G}=k\left(\frac{1}{\alpha}-\frac{1}{2} \mathrm{~S}\right) ; \quad \mathrm{H}=k\left(\frac{1}{\beta}-\frac{1}{2} \mathrm{~S}\right) ; \quad \mathrm{I}=k\left(\frac{1}{\gamma}-\frac{1}{2} \mathrm{~S}\right) .
$$

20. Using (43) and (47) in (19), we have

$$
\left.\begin{array}{rl}
\delta \mathrm{E}=\frac{1}{2} k\{ & -\frac{\delta \alpha}{\alpha^{2}}-\frac{\delta \beta}{\beta^{2}}-\frac{\delta \gamma}{\gamma^{2}}+\mathrm{S}\left(\frac{\delta \alpha}{\alpha}+\frac{\delta \beta}{\beta}+\frac{\delta \gamma}{\gamma}\right) \\
& +\frac{\delta \alpha^{2}}{\alpha^{3}}+\frac{\delta \beta^{2}}{\beta^{3}}+\frac{\delta \gamma^{2}}{\gamma^{3}}-\frac{1}{2} \mathrm{~S}\left(\frac{\delta \alpha^{2}}{\alpha^{2}}+\frac{\delta \beta^{2}}{\beta^{2}}+\frac{\delta \gamma^{2}}{\gamma^{2}}\right)
\end{array}\right\}
$$


Now we have, by $(2), \log (\alpha \beta \gamma)=0$. Hence, taking the variation of this as far as terms of the second order,

$$
\frac{\delta \alpha}{\alpha}+\frac{\delta \beta}{\beta}+\frac{\delta \gamma}{\gamma}-\frac{1}{2}\left(\frac{\delta \alpha^{2}}{\alpha^{2}}+\frac{\delta \beta^{2}}{\beta^{2}}+\frac{\delta \gamma^{2}}{\gamma^{2}}\right)=0 \text {. }
$$

which reduces $(48)$ to

$$
\delta \mathrm{E}=\frac{1}{2} k\left(-\frac{\delta \alpha}{\alpha^{2}}-\frac{\delta \beta}{\beta^{2}}-\frac{\delta \gamma}{\gamma^{2}}+\frac{\delta \alpha^{2}}{\alpha^{3}}+\frac{\delta \beta^{2}}{\beta^{3}}+\frac{\delta \gamma^{2}}{\gamma^{3}}\right) .
$$

Remembering that cubes and higher powers are to be neglected, we see that $(50)$ is equivalent to

$$
\delta \mathrm{E}=\frac{1}{2} k \delta\left(\frac{1}{\alpha}+\frac{1}{\beta}+\frac{1}{\gamma}\right) . \quad . \quad . \quad .
$$

Hence if we take $k$ constant, we have

$$
\mathrm{E}=\frac{1}{2} k\left(\frac{1}{\alpha}+\frac{1}{\beta}+\frac{1}{\gamma}-3\right) \quad . \quad . .
$$

and it is clear that $k$ must be stationary (that is to say, $\delta k=0$ ) for any particular values of $\alpha, \beta, \gamma$ for which (51) holds; and if (51) holds for all values, $k$ must be constant for all values of $\alpha, \beta, \gamma$.

21. Going back to (29), taking Q great enough to allow W/Q to be neglected, and simplifying by (46), (43), and $(44)$, we find

$$
\frac{\mathrm{W}}{\mathrm{Q}}=k\left(\frac{l^{2}}{\alpha}+\frac{m^{2}}{\beta}+\frac{n^{2}}{\gamma}\right) \int_{0}^{h} d p \sigma^{2} . . .
$$

and the problem $(\S 17)$ of determining $l, m, n$, subject to (5) and $(6)$, to make $l^{2} / \alpha+m^{2} / \beta+n^{2} / \gamma$ a maximum or minimum for given values of $\lambda, \mu, \nu$, yields the equations $\omega \lambda-\omega^{\prime} l+\frac{l}{\alpha}=0 ; \omega \mu-\omega^{\prime} m+\frac{m}{\beta}=0 ; \omega \nu-\omega^{\prime} n+\frac{n}{\gamma}=0 .(54)$, $\omega, \omega^{\prime}$ denoting indeterminate multipliers; whence

$$
\left.\begin{array}{l}
\omega^{\prime}=\frac{l^{2}}{\alpha}+\frac{m^{2}}{\beta}+\frac{n^{2}}{\gamma} \quad . . . . . . \\
\omega^{2}=l^{2}\left(\omega^{\prime}-\frac{1}{\alpha}\right)^{2}+m^{2}\left(\omega^{\prime}-\frac{1}{\beta}\right)^{2}+n^{2}\left(\omega^{\prime}-\frac{1}{\gamma}\right)^{2} \\
\omega \lambda=l\left(-\frac{1-l^{2}}{\alpha}+\frac{m^{2}}{\beta}+\frac{n^{2}}{\gamma}\right), \\
\omega \mu=m\left(\frac{l^{2}}{\alpha}-\frac{1-m^{2}}{\beta}+\frac{n^{2}}{\gamma}\right), \\
\omega \nu=n\left(\frac{l^{2}}{\alpha}+\frac{m^{2}}{\beta}-\frac{1-n^{2}}{\gamma}\right),
\end{array}\right\} . . . \quad
$$


These formulas are not directly convenient for finding $l, m, n$ from $\lambda, \mu, \nu$ given (the ordinary formulas for doing so need not be written here); but thev give $\lambda, \mu, v$ explicitly in terms of $l, m, n$ supposed known; that is to say, they solve the problem of finding the wave-front of the simple laminar wave whose direction of vibration is $(l, m, n)$. The velocity is given by

$$
v^{2}=k\left(\frac{l^{2}}{\alpha}+\frac{m^{2}}{\beta}+\frac{n^{2}}{\gamma}\right) . . . . . .
$$

It is interesting to notice that this depends solely on the direction of the line of vibration; and that (except in special cases, of partial or complete isotropy) there is just one wavefront for any given line of vibration. These are precisely in every detail the conditions of Fresnel's Kinematics of Double Refraction.

22. Going back to (35) and (36), let us see if we can fit them to double refraction with line of vibration in the plane of polarization. This would require (36) to be the ordinary ray, and therefore requires the fulfilment of (38), as did the other supposition; but instead of (37) we now have [in order to make (36) constant]

$$
\mathrm{A}=\mathrm{B}=\mathrm{C} \quad \cdot \quad \cdot \quad \cdot \quad \cdot \quad(59),
$$

and therefore each, in virtue of (14), zero ; and

$$
\alpha=\beta=\gamma=1 \text {; }
$$

so that we are driven to complete isotropy. Hence our present form $(\S 7)$ of the stress theory of double refraction cannot be fitted to give line of vibration in the plane of polarization. We have seen $(\$ 21)$ that it does give line of vibration perpendicular to the plane of polarization with exactly Fresnel's form of wave-surface, when fitted for the purpose, by the simple assumption that the potential energy of the strained solid is expressed by (52) with $k$ constant? It is important to remark that $k$ is the rigidity-modulus of the unstrained isotropic solid.

23. From (58) we see that the velocities of the waves corresponding to the three cases, $l=1, m=1, n=1$, respectively are $\sqrt{ }(k / \alpha), \sqrt{ }(k / \beta), \sqrt{ }(k / \gamma)$. Hence the velocity of any wave whose vibrations are parallel to any one of the three principal elongations, multiplied by this elongation, is equal to the velocity of a wave in the unstrained isotropic solid. 